\title{
A family of edge-transitive Cayley graphs
}

\author{
Jiangmin Pan ${ }^{1}$ - Zhaofei Peng1
}

Received: 6 April 2017 / Accepted: 15 March 2018 / Published online: 5 April 2018 char 169 Springer Science+Business Media, LLC, part of Springer Nature 2018

\begin{abstract}
Edge-transitive graphs of order a prime or a product of two distinct primes with any positive integer valency, and of square-free order with valency at most 7 have been classified by a series of papers. In this paper, a complete classification is given of edge-transitive Cayley graphs of square-free order with valency less than the smallest prime divisor of the order. This leads to new constructions of infinite families of both arc-regular Cayley graphs and edge-regular Cayley graphs (so half-transitive). Also, as by-products, it is proved that, for any given positive integers $k, s \geq 1$ and $m, n \geq 2$, there are infinitely many arc-regular normal circulants of valency $2 k$ and order a product of $s$ primes, and there are infinitely many edge-regular normal metacirculants of valency $2 m$ and order a product of $n$ primes; such arc-regular and edge-regular examples are also specifically constructed.
\end{abstract}

Keywords Edge-transitive graph · Half-transitive graph · Arc-regular graph · Edge-regular graph $\cdot$ Normal Cayley graph

Mathematics Subject Classification 20B15 - 20B30 - 05C25

This work was partially supported by National Natural Science Foundation of China (11461007, 11231008).

$\triangle$ Jiangmin Pan jmpan@ynu.edu.cn

1 School of Statistics and Mathematics, Yunnan University of Finance and Economics, Kunming, People's Republic of China 


\section{Introduction}

For a graph $\Gamma$, if its full automorphism group Aut $\Gamma$ is transitive on its vertex set, edge set or arc set, then $\Gamma$ is called vertex-transitive, edge-transitive or arc-transitive, respectively. An arc-transitive graph is edge-transitive. In particular, if Aut $\Gamma$ is regular on the arc set or the edge set of $\Gamma$, then $\Gamma$ is called arc-regular or edge-regular, respectively; if Aut $\Gamma$ is transitive on both vertex set and edge set of $\Gamma$, but intransitive on the arc set of $\Gamma$, then $\Gamma$ is called half-transitive.

Edge-transitive graphs of square-free order (namely, not divisible by any prime square) provide a rich source of many interesting families of graphs, and have a rich research history. In 1971, Chao [6] classified arc-transitive graphs of prime order $p$, then Cheng and Oxley [7] classified edge-transitive graphs of order $2 p$, and Wang and $\mathrm{Xu}$ [29] classified arc-transitive graphs of order $3 p$. These results were generalized to the case of order a product of two distinct primes by Praeger et al. [26,27]. Moreover, edge-transitive graphs of square-free order and valency at most 7 have been classified by $[9,17,19,20]$. Quite recently, Li et al. [18] characterized the 'basic' edge-transitive graphs (namely, each nontrivial normal subgroup of the edge-transitive automorphism group has at most two orbits on the vertex set) of square-free order, with certain cases needing further research. It seems difficult to approach a general classification of edgetransitive graphs of square-free order. Actually, the classification of edge-transitive graphs of order $6 p$ with $p$ a prime has not been reached yet.

A graph $\Gamma$ is called a Cayley graph of a group $R$ if there is a subset $S \subseteq R \backslash\{1\}$, with $S=S^{-1}:=\left\{s^{-1} \mid s \in S\right\}$, such that its vertex set equals $R$ and two vertices $g$ and $h$ are adjacent in $\Gamma$ if and only if $h g^{-1} \in S$. This Cayley graph is denoted by Cay $(R, S)$. For convenience, a Cayley graph of a cyclic group is also called a circulant, and a Cayley graph of a metacyclic group is also called a metacirculant. It is well known that a graph $\Gamma$ is isomorphic to a Cayley graph of a group $R$ if and only if Aut $\Gamma$ contains a subgroup which is isomorphic to $R$ and acts regularly on the vertex set of $\Gamma$, see [3, Proposition 16.3]. Let $\Gamma=\operatorname{Cay}(R, S)$. Then, the right regular presentation

$$
\hat{R}=\{\hat{g} \mid \hat{g}: x \mapsto x g, \text { for all } g, x \in R\}
$$

is a subgroup of Aut $\Gamma$, refer to [13]. If $\hat{R}$ is normal in an automorphism group $G \leq$ Aut $\Gamma$, then $\Gamma$ is called a $G$-normal Cayley graph of $R$. In particular, $\Gamma$ is called a normal Cayley graph of $R$ if $\hat{R}$ is normal in Aut $\Gamma$.

The purpose of this paper is to give a complete classification of edge-transitive Cayley graphs of square-free order with valency less than the smallest prime divisor of the order of the graph, which in particular leads to new constructions of infinite family of both arc-regular and edge-regular (so half-transitive) Cayley graphs. Also, by using the outcomes, the paper will prove two theorems regarding arc-regular and edge-regular normal Cayley graphs with any prescribed even valency.

To state our results, we need to introduce some terminologies and notations (which are standard) used in this paper. For example, for a positive integer $n$, we use $\mathbb{Z}_{n}, \mathrm{D}_{n}$ with $n$ even, $\mathrm{A}_{n}$ and $\mathrm{S}_{n}$ to denote the cyclic group and the dihedral group of order $n$, the alternating group and the symmetric graph of degree $n$, respectively, and use $\mathbb{Z}_{n}^{*}$ to denote the multiplicative group of the residue class ring modular $n$ (then $\mathbb{Z}_{n}^{*} \cong \operatorname{Aut}\left(\mathbb{Z}_{n}\right)$ 
is abelian). Given two groups $N$ and $H$, denote by $N \times H$ the direct product of $N$ and $H$, by $N . H$ an extension of $N$ by $H$, and if such an extension is split, then we write $N$ : $H$ instead of $N . H$. Also, denote by $\mathrm{K}_{n}$ the complete graph of order $n$, by $V \Gamma$ the vertex set of a graph $\Gamma$, by $\Gamma(\alpha)$ the set of vertices adjacent to the vertex $\alpha$ in $\Gamma$, and if $\Gamma$ is regular, denote by $\operatorname{val}(\Gamma)$ its valency.

The following is a complete classification of the edge-transitive Cayley graphs of our case. For convenience, certain graphs appearing in the theorem are introduced in Sect. 2.

Theorem 1.1 Let $\Gamma=\operatorname{Cay}(R, S)$ be a connected $G$-edge-transitive Cayley graph of square-free order $n$ with $\hat{R} \leq G \leq$ Aut $\Gamma$, and suppose the valency of $\Gamma$ is less than the smallest prime divisor of $n$. Then one of the following statements holds.

1. $R=\langle a\rangle \cong \mathbb{Z}_{n}$ is cyclic, and

$$
\Gamma=\operatorname{Cay}\left(R, a^{H}\right), \text { with } \tau \in H \leq \operatorname{Aut}(\langle a\rangle),
$$

where $\tau$ is an automorphism of $R$ mapping a to $a^{-1}$. Further, the following statements hold.

(i) If $n$ is a prime and $H \cong \mathbb{Z}_{n-1}$, then $\Gamma=\mathrm{K}_{n}$, $G=\mathbb{Z}_{n}: \mathbb{Z}_{\frac{n}{2}}$ or $\mathbb{Z}_{n}: \mathbb{Z}_{n-1}$, and Aut $\Gamma=\mathrm{S}_{n}$.

(ii) If $n$ is a prime and $H<\mathbb{Z}_{n-1}$ or $n$ is not a prime, then $\Gamma$ is an arc-regular normal Cayley graph of $R, \operatorname{val}(\Gamma)=|H|$, and

$$
\text { Aut } \Gamma=\hat{R}: H= \begin{cases}G, & \text { if } \tau \in G ; \\ G:\langle\tau\rangle \cong G: \mathbb{Z}_{2}, & \text { if } \tau \notin G .\end{cases}
$$

2. $R=\langle a\rangle \times(\langle b\rangle:\langle c\rangle) \cong \mathbb{Z}_{h} \times\left(\mathbb{Z}_{t}: \mathbb{Z}_{l}\right)$ such that the center $Z(\langle b\rangle:\langle c\rangle)=1$,

$$
\Gamma=\operatorname{Cay}\left(R,\left\{a c^{z},\left(a c^{z}\right)^{-1}\right\}^{K}\right) \text { with }(z, l)=1,
$$

and $\operatorname{val}(\Gamma)=2|K|$, where $K \leq \mathbb{Z}_{h}^{*} \times \mathbb{Z}_{t}^{*}$ is a subgroup of $\operatorname{Aut}(R)$. Further, Aut $\Gamma=G=\hat{R}: K$ and $\Gamma$ is an edge-regular (so half-transitive) normal Cayley graph of $R$ with the only exception: $(h, t, l)=(1,11,5)$ and $\operatorname{val}(\Gamma)=4$.

3. $R \cong \mathbb{Z}_{11}: \mathbb{Z}_{5}, G \cong \mathbb{Z}_{11}: \mathbb{Z}_{10}$, Aut $\Gamma \cong \operatorname{PGL}(2,11)$, and $\Gamma$ is arc-transitive of valency 4 .

4. $G$ is almost simple, and $R, G, \operatorname{val}(\Gamma)$ and Aut $\Gamma$ are listed in Table 1 , where $G\left(q^{d}\right)$ is a regular subgroup of $\Gamma \mathrm{L}\left(1, q^{d}\right), P_{1}$ is a parabolic subgroup of $\operatorname{PSL}(d, q)$, $q=p^{e}, p$ is a prime and $\alpha \in V \Gamma$.

5. Either

(i) $G \cong\left(\mathrm{D}_{2 m} \times \operatorname{PSL}(2,23)\right)$.o with $o \leq \mathbb{Z}_{2}$, and $\Gamma \cong \mathrm{C}_{m} \times \Sigma$ is of valency 8 , where $m \mid n$ with the smallest prime divisor at least $13, \mathrm{C}_{m}$ is a cycle of length $m$, and $\Sigma$ satisfies Row 10 of Table 1 with valency 4; or

(ii) $G \cong(M \times \operatorname{PSL}(2,59))$.o with $o \leq \mathbb{Z}_{2}$, and $\Gamma \cong \Delta \times \Sigma$, where $(\Delta, M)$ (as $(\Gamma, G)$ there) satisfies part (1) or (2) with $\operatorname{val}(\Delta)=2$ or 4 , and $\Sigma$ satisfies Row 11 of Table 1 such that $\operatorname{val}(\Delta) \cdot \operatorname{val}(\Sigma)$ is less than the smallest prime divisor of $|V \Delta|$ and 29. 


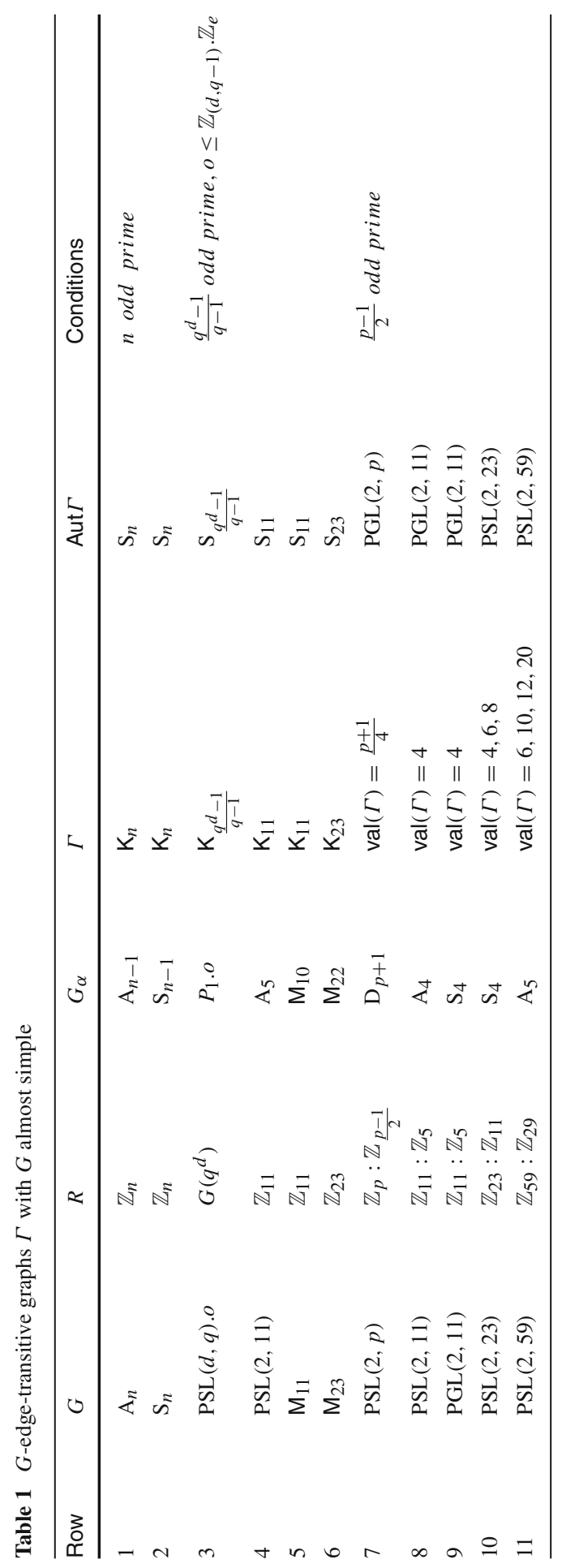


Remark For an automorphism $\sigma \in \operatorname{Aut}(R)$, the Cayley graphs $\operatorname{Cay}(R, S)$ and Cay $\left(R, S^{\sigma}\right)$ are isomorphic. A Cayley graph $\Gamma=\operatorname{Cay}(R, S)$ is called a CI-graph ('CI' stands for Cayley Isomorphism) if, whenever Cay $(R, T) \cong \operatorname{Cay}(R, S)$, we have $T=S^{\sigma}$ for some automorphism $\sigma \in \operatorname{Aut}(R)$. By [15, Theorem 4.1], if $R$ is of odd order and $\left(|R|, \mid(\text { Aut } \Gamma)_{\alpha} \mid\right)=1$, where $\alpha$ is a vertex of $\Gamma$, then $\Gamma$ is a CI-graph. Therefore, graphs in Theorem 1.1 are CI-graphs, and consequently, one may enumerate the non-isomorphic arc-regular and edge-regular Cayley graphs of order $n$ appearing in parts (1) and (2) of Theorem 1.1.

A graph $\Gamma$ is called locally primitive if the vertex stabilizer (Aut $\Gamma)_{\alpha}$ is primitive on $\Gamma(\alpha)$ for each vertex $\alpha$ of $\Gamma$. Locally primitive graphs are edge-transitive. For a positive integer $s$, an $s$-arc of a graph $\Gamma$ is a sequence $v_{0}, v_{1}, \ldots, v_{s}$ of $s+1$ vertices such that $v_{i-1}, v_{i}$ are adjacent for $1 \leq i \leq s$ and $v_{i-1} \neq v_{i+1}$ for $1 \leq i \leq s-1$. If Aut $\Gamma$ is transitive on the set of $s$-arcs of $\Gamma$, but not transitive on the set of $(s+1)$-arcs, then $\Gamma$ is simply called $s$-transitive.

Theorem 1.1 has the following corollary which shows that the connected locally primitive Cayley graphs of odd square-free order with 'small' valency are rare.

Corollary 1.2 Let $\Gamma$ be a connected locally primitive Cayley graph of square-free order $n$. Suppose further the valency of $\Gamma$ is less than the smallest prime divisor of $n$. Then $\Gamma$ is either 1-transitive or 2-transitive, and further, the triple $(\Gamma, n$, Aut $\Gamma)$ is listed in Table 2 for $\alpha \in V \Gamma$.

By definition, half-transitive graphs, arc-regular graphs, edge-regular graphs and normal Cayley graphs are closely related to their full automorphism groups. Since determining the full automorphism groups (generally very difficult) of given graphs is one of the fundamental topics in the field of algebraic graph theory, constructing each of the above classes of graphs is an interesting topic in the literature. For arc-regular graphs, connected ones with valency 2 are just cycles, quite a lot is now known are with small valency (especially valency 3 and 4), see [2,11,12,21,23] for examples. For any even valency, there is an infinite family of arc-regular graphs of order a prime with cyclic stabilizer by Chao [6] and independently by Alspach [1].

Part (1)(ii) of Theorem 1.1 provides a new infinite family of arc-regular normal circulants of square-free order with abelian vertex stabilizer. We may actually further prove the following theorem.

Table 2 Locally primitive Cayley graphs $\Gamma$

\begin{tabular}{llllll}
\hline$\Gamma$ & $n$ & Aut $\Gamma$ & $(\text { Aut } \Gamma)_{\alpha}$ & Transitivity & Conditions \\
\hline $\mathrm{K}_{n}$ & $n$ & $\mathrm{~S}_{n}$ & $\mathrm{~S}_{n-1}$ & 2 -transitive & n odd prime \\
$\operatorname{val}(\Gamma)=4$ & 55 & $\operatorname{PGL}(2,11)$ & $\mathrm{S}_{4}$ & 2 -transitive & \\
$\operatorname{val}(\Gamma)=4$ & 253 & $\operatorname{PSL}(2,23)$ & $\mathrm{S}_{4}$ & 2 -transitive & \\
$\operatorname{val}(\Gamma)=6,10$ & 3481 & $\operatorname{PSL}(2,59)$ & $\mathrm{A}_{5}$ & 2 -transitive & \\
$\operatorname{val}(\Gamma)=\frac{p+1}{4}$ & $\frac{p(p-1)}{2}$ & $\operatorname{PGL}(2, p)$ & $\mathrm{D}_{p+1}$ & $1-$ transitive & $\frac{p-1}{2}, \frac{p+1}{4}$ primes \\
\hline
\end{tabular}


Theorem 1.3 For any given positive integers $k$ and $s$, there are infinitely many arcregular normal circulants of valency $2 k$ and order a product of $s$ primes.

We remark that the proof of Theorem 1.3 includes a specific construction of such arc-regular circulants, see Construction 7.2 below.

The known constructions of edge-regular Cayley graphs are very few. Clearly, edge-regular Cayley graphs are half-transitive, but the inverse is generally not true. The study of half-transitive graphs was initiated by a question of Tutte [30] regarding the existence of half-transitive graphs with even valency, after he proved that a vertextransitive and edge-transitive graph with odd valency is arc-transitive. Since connected graphs with valency 2 are cycles, which are arc-transitive, the valency of half-transitive graphs must be even and at least 4. Quite a lot known examples of half-transitive graphs are in the 4-valent case, see $[4,8,22,23]$ for references.

Motivated by Theorem 1.3, it is natural to ask: Are there infinitely many edgeregular Cayley graphs with any even valency at least 4 ? The following theorem answers it affirmatively and the corresponding examples are constructed (see Construction 7.3 below). Note that edge-transitive Cayley graphs of abelian groups are arc-transitive and so never be edge-regular.

Theorem 1.4 For any given positive integers $k, t \geq 2$, there are infinitely many edgeregular normal metacirculants (so half-transitive) of valency $2 k$ and order a product of $t$ primes.

After this introductory section, some preliminary results are given in Sect. 2, and two technical lemmas regarding the normal subgroups of the edge-transitive automorphism groups are proved in Sect. 3. Then, by investigating the soluble group case and the almost simple group case in Sects. 4 and 5, we finish the proofs of Theorem 1.1 and Corollary 1.2 in Sect. 6. Finally, in Sect. 7, we present proofs of Theorems 1.3 and 1.4 .

\section{Preliminaries}

\subsection{Some background results}

For a group $G$, the Fitting subgroup of $G$ is the largest nilpotent normal subgroup of $G$. Clearly, the Fitting subgroup is a characteristic subgroup. The following is a basic property of the Fitting subgroups of soluble groups.

Lemma 2.1 [28, P. 30, Corollary] Let $G$ be a soluble group and $\mathrm{F}$ the Fitting subgroup of $G$. Then $\mathrm{F} \neq 1$ and the centralizer $C_{G}(\mathrm{~F}) \leq \mathrm{F}$.

Given a group $G$ and its subgroup $H$, let $[G: H]=\{H g \mid g \in G\}$, the set of right cosets of $H$ in $G$. The coset action of $G$ on $[G: H]$ is defined by:

$$
(H g)^{x}=H g x, \text { for all } g, x \in G .
$$

This coset action is faithful if and only if $\cap_{g \in G} H^{g}=1$ (refer to [10, Example 1.3.4]). In this case, $H$ is called core-free in $G$. 
Suppose $\sigma$ and $\tau$ are two actions of a group $G$ on sets $\Omega$ and $\Delta$, respectively. These actions are permutation equivalent if there is a bijection $\lambda: \Omega \rightarrow \Delta$ such that

$$
\lambda\left(\alpha^{\sigma(g)}\right)=(\lambda(\alpha))^{\tau(g)}, \text { for all } \alpha \in \Omega \text { and } g \in G
$$

It is well known that each transitive action of a group $G$ is permutation equivalent to the coset action of $G$ on $\left[G: G_{\alpha}\right]$. The following observation will be used in the later discussion.

Lemma 2.2 Let $G=H_{1} \times \cdots \times H_{S}$ be a group acting transitively on a set $\Omega$, with $s \geq 2$, such that $G_{\alpha}=\left(H_{1}\right)_{\alpha} \times \cdots \times\left(H_{s}\right)_{\alpha}$ for $\alpha \in \Omega$. Set $\Omega_{i}=\left[H_{i}:\left(H_{i}\right)_{\alpha}\right]$ for $i=1,2, \ldots, s$. Then, the action of $G$ on $\Omega$ is permutation equivalent to the following 'product action' of $G$ on the cartesian product $\Omega_{1} \times \cdots \times \Omega_{s}$ :

$$
\left(\left(H_{1}\right)_{\alpha} g_{1}, \ldots,\left(H_{s}\right)_{\alpha} g_{s}\right)^{x_{1} x_{2} \cdots x_{s}}=\left(\left(H_{1}\right)_{\alpha} g_{1} x_{1}, \ldots,\left(H_{s}\right)_{\alpha} g_{s} x_{s}\right)
$$

where $g_{i}, x_{i} \in H_{i}$ for $i=1,2, \ldots, s$.

Actually, we may identify the action of $G$ on $\Omega$ with the coset action of $G$ on $\left[G: G_{\alpha}\right]$. By assumption, any elements $g, x \in G$ can be uniquely expressed as $g=g_{1} g_{2} \cdots g_{s}$ and $x=x_{1} x_{2} \cdots x_{s}$ with $g_{i}, x_{i} \in H_{i}$. Then, it is easy to show that

$$
\lambda: G_{\alpha} g_{1} g_{2} \cdots g_{s} \rightarrow\left(\left(H_{1}\right)_{\alpha} g_{1},\left(H_{2}\right)_{\alpha} g_{2}, \ldots,\left(H_{s}\right)_{\alpha} g_{s}\right)
$$

is a bijection of $\left[G: G_{\alpha}\right]$ to $\Omega_{1} \times \cdots \times \Omega_{s}$ ensuring the equivalence.

Taking normal quotient graphs is a typical method for studying vertex-transitive graphs. Suppose $\Gamma$ is a $G$-vertex-transitive graph, where $G \leq$ Aut $\Gamma$ has an intransitive normal subgroup $N$. Denote by

$$
V \Gamma_{N}:=\left\{\alpha^{N} \mid \alpha \in V \Gamma\right\}
$$

the set of $N$-orbits in $V \Gamma$. The normal quotient graph $\Gamma_{N}$ of $\Gamma$ induced by $N$ is defined with vertex set $V \Gamma_{N}$ and two vertices $B, C \in V \Gamma_{N}$ are adjacent if and only if some vertex in $B$ is adjacent in $\Gamma$ to some vertex in $C$. If $\operatorname{val}(\Gamma)=\operatorname{val}\left(\Gamma_{N}\right)$, then $\Gamma$ is called a normal cover (or regular cover) of $\Gamma_{N}$.

The truth of the following lemma is easily justified.

Lemma 2.3 Let $\Gamma$ be a connected $G$-vertex-transitive and $G$-edge-transitive graph, where $G \leq$ Aut $\Gamma$ has an intransitive normal subgroup $N$. Then, the following statements hold:

1. $\Gamma_{N}$ is a connected $G / K$-edge-transitive graph, where $K$ is the kernel of $G$ acting on $V \Gamma_{N}$.

2. $K \leq \prod_{B \in V \Gamma_{N}} K^{B}$.

3. If $\Gamma$ is a normal cover of $\Gamma_{N}$, then $N$ is semi-regular on $V \Gamma$. 


\subsection{Normal Cayley graphs}

For a Cayley graph $\Gamma=\operatorname{Cay}(R, S)$, then $\hat{R}$ and

$$
\operatorname{Aut}(R, S)=\left\{\sigma \in \operatorname{Aut}(R) \mid S^{\sigma}=S\right\}
$$

are subgroups of $\operatorname{Aut} \Gamma$ (note that $\operatorname{Aut}(R, S)$ is also a subgroup of the automorphism group Aut $(R))$. Further, the following nice property holds.

Lemma 2.4 [13, Lemma 2.1] Let $\Gamma=\operatorname{Cay}(R, S)$ be a Cayley graph. Then, the normalizer $N_{\text {Aut } \Gamma}(\hat{R})=\hat{R}: \operatorname{Aut}(R, S)$.

Now, suppose $\Gamma=\operatorname{Cay}(R, S)$ is a $G$-normal edge-transitive Cayley graph, where $\hat{R} \triangleleft G \leq \operatorname{Aut} \Gamma$. By Lemma $2.4, G \leq \hat{R}: \operatorname{Aut}(R, S)$, and so $G_{1} \leq \operatorname{Aut}(R, S)$ has at most two orbits on the neighbor set $\Gamma(\mathbf{1})=S$, where $\mathbf{1}$ denotes the vertex of $\Gamma$ corresponding to the identity element of $R$. Observing that, for $s \in S$, if there is $g \in G_{\mathbf{1}}$ such that $s^{g}=s^{-1}$, then $(\mathbf{1}, s)^{g \hat{s}}=\left(\mathbf{1}, s^{-1}\right)^{\hat{s}}=(s, \mathbf{1})$, it follows that $\Gamma$ is $G$-arc-transitive. This suggests the following construction of normal edge-transitive Cayley graphs.

Construction 2.5 Let $R$ be a group. Take $H \leq \operatorname{Aut}(R)$ and $s \in R \backslash\{1\}$. Let

$$
S=\left\{s^{H},\left(s^{-1}\right)^{H}\right\} \text {, and } \Gamma=\operatorname{Cay}(R, S) \text {. }
$$

Then $\Gamma$ is a $G$-normal edge-transitive Cayley graph, where $G=\hat{R}: H$. Further, $\Gamma$ is $G$-half-transitive if $s^{-1} \notin s^{H}$, and $\Gamma$ is $G$-arc-transitive if $s^{-1} \in s^{H}$. In particular, $\Gamma$ is connected if and only if $\left\langle s^{H}\right\rangle=R$.

Conversely, each normal edge-transitive Cayley graph can be constructed as above.

Construction 2.5 provides a method to construct 'many' normal edge-transitive Cayley graphs. However, it is generally difficult to know whether the graphs are really normal Cayley graphs or not, due to the difficulty for finding their full automorphism groups.

\subsection{Product graphs}

Let $\Delta_{1}, \Delta_{2}, \ldots, \Delta_{s}$ be graphs with $s \geq 2$. Define the product graph $\Delta_{1} \times \Delta_{2} \times \cdots \times \Delta_{s}$ with vertex set $V \Delta_{1} \times V \Delta_{2} \times \cdots \times V \Delta_{s}$, and two vertices $u=\left(u_{1}, u_{2}, \ldots, u_{s}\right)$ and $v=\left(v_{1}, v_{2}, \ldots, v_{s}\right)$ are adjacent if and only if $u_{i}$ is adjacent to $v_{i}$ in $\Delta_{i}$ for each $i=1,2, \ldots, s$.

By definition, it is easy to verify the following lemma, see [14] for more properties of edge-transitive product graphs.

Lemma 2.6 The following statements hold:

1. $\operatorname{val}\left(\Delta_{1} \times \Delta_{2} \times \cdots \times \Delta_{S}\right)=\operatorname{val}\left(\Delta_{1}\right) \cdot \operatorname{val}\left(\Delta_{2}\right) \cdots \operatorname{val}\left(\Delta_{S}\right)$. 
2. Aut $\left(\Delta_{1}\right) \times \operatorname{Aut}\left(\Delta_{2}\right) \times \cdots \times \operatorname{Aut}\left(\Delta_{S}\right) \leq \operatorname{Aut}\left(\Delta_{1} \times \Delta_{2} \times \cdots \times \Delta_{S}\right)$.

3. If each $\Delta_{i}$ is vertex-transitive and edge-transitive, and at most one of $\Delta_{1}, \Delta_{2}, \ldots$, $\Delta_{S}$ is half-transitive, then $\Delta_{1} \times \Delta_{2} \times \cdots \times \Delta_{s}$ is edge-transitive.

Edge-transitive graphs which are vertex-transitive can be constructed as fusedorbital graphs, stated as following. For a transitive permutation group $G$ on a set $\Omega$, an orbital of $G$ is a digraph with vertex set $\Omega$ and arc set $(\alpha, \beta)^{G}$ where $\alpha, \beta \in \Omega$. If $(\alpha, \beta)^{G}=(\beta, \alpha)^{G}$, this orbital is called self-paired. A fused-orbital of $G$ is a graph with vertex set $\Omega$ and arc set $(\alpha, \beta)^{G} \cup(\beta, \alpha)^{G}$. If $(\alpha, \beta)^{G}=(\beta, \alpha)^{G}$, this fusedorbital is a self-paired orbital and is $G$-arc-transitive; if $(\alpha, \beta)^{G} \neq(\beta, \alpha)^{G}$, then the fused-orbital is a $G$-half-transitive graph. Conversely, it is easy to know that each vertex-transitive and edge-transitive graph can be reconstructed in this way.

The following result is due to Higman, see [10, Theorem 3.2A].

Lemma 2.7 Let $G$ be a transitive permutation group. Then, $G$ is primitive if and only if each nontrivial orbital is connected.

Lemma 2.8 Let $\Gamma$ be a $G$-vertex-transitive and $G$-edge-transitive graph, where $G \leq$ Aut $\Gamma$. Suppose further $G=H_{1} \times H_{2} \times \cdots \times H_{l}$ and $G_{\alpha}=\left(H_{1}\right)_{\alpha} \times\left(H_{2}\right)_{\alpha} \times \cdots \times\left(H_{l}\right)_{\alpha}$, where $l \geq 2$ and $\alpha \in V \Gamma$. Then

$$
\Gamma \cong \Gamma_{1} \times \Gamma_{2} \times \cdots \times \Gamma_{l},
$$

where $\Gamma_{i}$ is a fused-orbital graph of $H_{i}$ (so $H_{i}$-edge-transitive) with vertex stabilizer $\left(H_{i}\right)_{\alpha}$ for $i=1,2, \ldots, l$.

Proof Let $\Omega_{i}=\left[H_{i}:\left(H_{i}\right)_{\alpha}\right]$ for $i=1,2, \ldots, l$. Since $G \leq \operatorname{Aut} \Gamma, G_{\alpha}$ is core-free in $G$, it follows that $\left(H_{i}\right)_{\alpha}$ is core-free in $H_{i}$, so $H_{i}$ can be viewed as a transitive permutation group on $\Omega_{i}$ by coset action. By Lemma 2.2, we may identify $V \Gamma$ with $\Omega_{1} \times \Omega_{2} \times \cdots \times \Omega_{l}$, and identify the action of $G$ on $V \Gamma$ with the product action of $G$ on $\Omega_{1} \times \Omega_{2} \times \cdots \times \Omega_{l}$.

Now since $\Gamma$ is a $G$-vertex-transitive and $G$-edge-transitive graph, $\Gamma$ is a fusedorbital graph of $G$ on $V \Gamma$, with arc set $(\alpha, \beta)^{G} \cup(\beta, \alpha)^{G}$ say, where $\beta \in \Gamma(\alpha)$. By the discussion above, we may label $\alpha=\left(\alpha_{1}, \alpha_{2}, \ldots, \alpha_{l}\right)$ and $\beta=\left(\beta_{1}, \beta_{2}, \ldots, \beta_{l}\right)$ with $\alpha_{i}, \beta_{i} \in \Omega_{i}$. Then $G_{\alpha}=\left(H_{1}\right)_{\alpha_{1}} \times\left(H_{2}\right)_{\alpha_{2}} \times \cdots \times\left(H_{s}\right)_{\alpha_{l}}$.

First suppose $G$ is arc-transitive on $\Gamma$. Then $G_{\alpha}$ is transitive on $\Gamma(\alpha)$, so

$$
\Gamma\left(\alpha_{1}, \alpha_{2}, \ldots, \alpha_{l}\right)=\left(\beta_{1}, \beta_{2}, \ldots, \beta_{l}\right)^{G_{\alpha}}=\left(\beta_{1}^{\left(H_{1}\right)_{\alpha_{1}}}, \beta_{2}^{\left(H_{2}\right)_{\alpha_{2}}}, \ldots, \beta_{l}^{\left(H_{l}\right)_{\alpha_{l}}}\right) .
$$

It follows $\Gamma \cong \Gamma_{1} \times \Gamma_{2} \times \cdots \times \Gamma_{l}$, where $\Gamma_{i}$ is a self-paired orbital graph of $H_{i}$ on $\Omega_{i}$ with $\operatorname{arc} \operatorname{set}\left(\alpha_{i}, \beta_{i}\right)^{H_{i}}$. If $G$ is half-transitive on $\Gamma$, then $G_{\alpha}$ has two orbits on $\Gamma(\alpha)$, that is $\Gamma(\alpha)=\beta^{G_{\alpha}} \cup \gamma^{G_{\alpha}}$ for some $\beta, \gamma \in \Gamma(\alpha)$. Then one may similarly prove $\Gamma \cong \Gamma_{1} \times \Gamma_{2} \times \cdots \times \Gamma_{l}$, with each $\Gamma_{i}$ a fused-orbital graph of $H_{i}$ on $\Omega_{i}$. 


\section{Normal subgroups}

From now on, we begin the process for proving Theorem 1.1. For convenience, in Sects. 3-6 of this paper, we always make the following hypothesis (the notations used in the hypothesis are also fixed).

Hypothesis 3.1 Let $\Gamma=\mathrm{Cay}(R, S)$ be a connected $G$-edge-transitive Cayley graph of square-free order $n$, where $\hat{R} \leq G \leq$ Aut $\Gamma$ and $n=p_{1} p_{2} \ldots p_{s}$, with primes $p_{1}<p_{2}<\cdots<p_{s}$. Suppose further $k:=\operatorname{val}(\Gamma)<p_{1}$. Set $\pi=\left\{p_{1}, p_{2}, \ldots, p_{s}\right\}$.

Then $R$ is of square-free order $n$ and so is a metacyclic group, and $n$ is odd, hence $k$ is even since an edge-transitive graph of odd order must has even valency. For $\alpha \in V \Gamma$, denote by $G_{\alpha}^{\Gamma(\alpha)}$ the induced permutation group of the action of $G_{\alpha}$ on $\Gamma(\alpha)$. Then $G_{\alpha}^{\Gamma(\alpha)} \leq \mathrm{S}_{k}$. Since $\Gamma$ is connected and $G$-vertex-transitive, $\left|G_{\alpha}\right|$ and $\left|G_{\alpha}^{\Gamma(\alpha)}\right|$ have the same prime divisors (see [6]), so each prime divisor of $\left|G_{\alpha}\right|$ is less then $p_{1}$.

As usual, for a group $H$ and a prime $p|| H \mid$, we use $H_{p}$ to denote a Sylow $p$-subgroup of $H$. Moreover, if $|H|=r_{1}^{t_{1}} r_{2}^{t_{2}} \cdots r_{l}^{t_{l}}$ is a prime factorization, let

$$
|H|_{\pi}=\prod_{r_{i} \in \pi} r_{i}^{t_{i}}, \quad|H|_{\pi^{\prime}}=\prod_{r_{i} \notin \pi} r_{i}^{t_{i}} .
$$

Because $\left|G: G_{\alpha}\right|=n$, we obtain $|G|_{\pi}=n=p_{1} p_{2} \cdots p_{s}$ and $\left|G_{\alpha}\right|=|G|_{\pi^{\prime}}$.

The next lemma will be repeatedly used in the subsequent discussion.

Lemma 3.1 The normal subgroups of $G$ have the following properties, where $\alpha \in$ $V \Gamma$.

1. Each minimal normal subgroup of $G$ is simple.

2. If $1 \neq N \triangleleft G$, then $\left|N_{\alpha}\right|=|N|_{\pi^{\prime}},|N \cap \hat{R}|=\left|\alpha^{N}\right|=\left|N: N_{\alpha}\right|=|N|_{\pi} \neq 1$, and $N \cap \hat{R}$ is regular on $\alpha^{N}$. In particular, if $N$ is transitive on $V \Gamma$, then $\hat{R} \leq N$.

3. Suppose $M, N \triangleleft G$ such that $M \cap N=1$. Then $(|M|,|N|, n)=1$ and $(\mid M$ : $\left.M_{\alpha}|| N:, N_{\alpha} \mid\right)=1$.

4. Suppose $X=N_{1} \times N_{2} \times \cdots \times N_{l}$, where $N_{i} \triangleleft G$ for $i=1,2, \ldots, l$. Then $X_{\alpha}=\left(N_{1}\right)_{\alpha} \times\left(N_{2}\right)_{\alpha} \times \cdots \times\left(N_{l}\right)_{\alpha}$.

Proof 1 . Let $M$ be a minimal normal subgroup of $G$. Then $M \cong T^{d}$ with $T$ a simple group and $d \geq 1$. Since $\left|\alpha^{M}\right|=\left|M: M_{\alpha}\right| \neq 1$ and $\left|\alpha^{M}\right|$ divides $|V \Gamma|$ with $|V \Gamma|=n$, we have $|M|_{\pi} \neq 1$. Then, as $|M|_{\pi}=\left(|T|_{\pi}\right)^{d}$ divides $|G|_{\pi}=$ $p_{1} p_{2} \cdots p_{s}$, we obtain $d=1$ and $M$ is simple.

2. Since $N \neq 1$ is normal in $G,\left|N: N_{\alpha}\right|=\left|\alpha^{N}\right| \neq 1$ divides $n$, and since $N_{\alpha} \leq G_{\alpha}$ is a $\pi^{\prime}$-subgroup, we have $\left|\alpha^{N}\right|=|N|_{\pi}$ and $\left|N_{\alpha}\right|=|N|_{\pi^{\prime}}$. Let $Y=N \hat{R}$. Then $|Y|=\frac{|N||\hat{R}|}{|N \cap \hat{R}|}$ divides $|G|$, so $|N|_{\pi}=|N \cap \hat{R}|_{\pi}=|N \cap \hat{R}|$ and $N \cap \hat{R}$ is regular on $\alpha^{N}$. Further, if $N$ is transitive on $V \Gamma$, then $|N \cap \hat{R}|=|N|_{\pi}=n=|\hat{R}|$, so $\hat{R} \leq N$.

3. Now $M N=M \times N \triangleleft G$. Then, since $|M|_{\pi}|N|_{\pi}$ divides $|G|_{\pi}=p_{1} p_{2} \cdots p_{s}$, $(|M|,|N|, n)=\left(|M|_{\pi},|N|_{\pi}\right)=1$, and by (2), $\left(\left|M: M_{\alpha}\right|,\left|N: N_{\alpha}\right|\right)=1$. 
4. Clearly $X_{\alpha} \geq\left(N_{1}\right)_{\alpha} \times\left(N_{2}\right)_{\alpha} \times \cdots \times\left(N_{l}\right)_{\alpha}$. On the other hand, part (2) implies

$$
\left|X_{\alpha}\right|=|X|_{\pi^{\prime}}=\left|N_{1}\right|_{\pi^{\prime}}\left|N_{2}\right|_{\pi^{\prime}} \cdots\left|N_{t}\right|_{\pi^{\prime}}=\left|\left(N_{1}\right)_{\alpha}\right|\left|\left(N_{2}\right)_{\alpha}\right| \cdots\left|\left(N_{l}\right)_{\alpha}\right|,
$$

hence $X_{\alpha}=\left(N_{1}\right)_{\alpha} \times\left(N_{2}\right)_{\alpha} \times \cdots \times\left(N_{l}\right)_{\alpha}$.

Let $T=\operatorname{PSL}\left(d, p^{e}\right)$ be a nonabelian simple group with $d \geq 2$ and $p$ a prime. It is known that $T$ has a natural 2-transitive permutation representation on the set of projective points (1 dimensional subspaces) of $d$-dimensional linear space over $p^{e}$-element field, with degree $\frac{p^{d e}-1}{p^{e}-1}$ and vertex stabilizer

$$
P_{1}:= \begin{cases}\mathbb{Z}_{p}^{e}: \mathbb{Z}_{\frac{p^{e}-1}{\left(2, p^{e}-1\right)},}, & \text { if } d=2 \\ \mathbb{Z}_{p}^{e(d-1)} \cdot \mathbb{Z}_{\frac{p^{e}-1}{\left(d, p^{e}-1\right)}} \cdot \operatorname{PSL}\left(d-1, p^{e}\right) \cdot \mathbb{Z}_{\left(d-1, p^{e}-1\right),}, & \text { if } d \geq 3\end{cases}
$$

Also, the outer automorphism group

$$
\operatorname{Out}(T)= \begin{cases}\mathbb{Z}_{\left(2, p^{e}-1\right)} \times \mathbb{Z}_{e}, & \text { if } d=2 \\ \mathbb{Z}_{\left(d, p^{e}-1\right)} \cdot \mathbb{Z}_{e} \cdot \mathbb{Z}_{2}, & \text { if } d \geq 3\end{cases}
$$

In particular, $\mid$ Out $(T) \mid \leq 2 e\left(d, p^{e}-1\right)$.

Lemma 3.2 Let $T$ be a nonabelian simple normal subgroup of $G$, and suppose that $T$ is intransitive on $V \Gamma$ and $p_{1} \leq|\operatorname{Out}(T)|$. Then $\left(T, T_{\alpha}\right) \neq\left(\operatorname{PSL}\left(d, p^{e}\right), P_{1}\right)$ for $\alpha \in V \Gamma$.

Proof Since $\Gamma$ is connected and $T$ is intransitive on $V \Gamma$, there exist two $T$-orbits $B_{1}$ and $B_{2}$ such that the induced subgraph $\left[B_{1}, B_{2}\right]$ is not an empty graph (recall that the graph $\left[B_{1}, B_{2}\right]$ is defined with vertex set $B_{1} \cup B_{2}$ and edge set consisting of edges of $\Gamma$ between $B_{1}$ and $B_{2}$ ). Since $T$ is transitive on $B_{1}$ and $B_{2},\left[B_{1}, B_{2}\right]$ is a regular graph.

If $\operatorname{val}\left(\left[B_{1}, B_{2}\right]\right)=1$, then $\operatorname{val}(\Gamma)=\operatorname{val}\left(\Gamma_{T}\right), \Gamma$ is a normal cover of $\Gamma_{T}$. By Lemma 2.3(3), $T$ is semi-regular on $V \Gamma$ and so $|T| \mid n$, which is a contradiction. Thus, val $\left(\left[B_{1}, B_{2}\right]\right) \geq 2$. Let $\alpha \in B_{1}$.

Suppose, on the contrary, $\left(T, T_{\alpha}\right)=\left(\operatorname{PSL}\left(d, p^{e}\right), P_{1}\right)$. Then $T$ is 2-transitive on $B_{i}$ with degree $\left|B_{i}\right|=\left|T: T_{\alpha}\right|=\frac{p^{d e}-1}{p^{e}-1}$ for $i=1,2$. Noting that $\operatorname{PSL}\left(d, p^{e}\right)$ has unique 2-transitive permutation representation of degree $\frac{p^{d e}-1}{p^{e}-1}$ if $d=2$, and has exactly two 2-transitive permutation representations of degree $\frac{p^{d e}-1}{p^{e}-1}$ if $d \geq 3$, namely, one is on the set of projective points and the another is on the set of hyperplanes $((d-1)$ dimensional subspaces), see [5, Theorem $5.3(\mathrm{~S})]$.

Assume first $T^{B_{1}}$ and $T^{B_{2}}$ are permutation equivalent. Then $T_{\alpha}$ fixes a vertex $\beta \in B_{2}$ and so $T_{\alpha}=T_{\beta}$ as they have the same order, hence $T_{\alpha}=T_{\beta}$ is transitive on $B_{2} \backslash\{\beta\}$ due to the 2-transitivity of $T$ on $B_{2}$. If $\beta \in \Gamma(\alpha)$, then val $\left(\left[B_{1}, B_{2}\right]\right)=1$, a contradiction. Hence there is a vertex $\gamma \in B_{2} \backslash\{\beta\}$ which is adjacent to $\alpha$. As $T_{\alpha}$ is transitive on $B_{2} \backslash\{\beta\}$, it follows $\left[B_{1}, B_{2}\right] \cong \mathrm{K}_{\left|B_{1}\right|,\left|B_{1}\right|}-\left|B_{1}\right| \mathrm{K}_{2}$, and so

$$
\operatorname{val}(\Gamma) \geq \operatorname{val}\left(\left[B_{1}, B_{2}\right]\right)=\left|B_{1}\right|-1=\frac{p^{e d}-1}{p^{e}-1}-1=\frac{p^{e}\left(p^{e(d-1)}-1\right)}{p^{e}-1} .
$$


By assumption, we obtain

$$
\frac{p^{e}\left(p^{e(d-1)}-1\right)}{p^{e}-1} \leq \operatorname{val}(\Gamma)<p_{1} \leq|\mathrm{Out}(T)| \leq 2 e\left(d, p^{e}-1\right),
$$

which is not possible.

Assume now $T^{B_{1}}$ and $T^{B_{2}}$ are permutation inequivalent. Then, $B_{1}$ and $B_{2}$ can be identified with the set of projective points and the set of hyperplanes, respectively, and the graph $\left[B_{1}, B_{2}\right]$ is isomorphic to the point-hyperplane incidence graph or pointhyperplane non-incidence graph, see [24, p. 193] or [25]. Thus, if there is a hyperplane $\gamma \in \Gamma(\alpha)$ such that $\alpha$ is contained in $\gamma$, then val $\left(\left[B_{1}, B_{2}\right]\right)=\frac{p^{e(d-1)}-1}{p^{e}-1}$, the number of 1-subspaces of a $(d-1)$-subspace; if each hyperplane $\gamma \in \Gamma(\alpha)$ does not contain $\alpha$, then $\operatorname{val}\left(\left[B_{1}, B_{2}\right]\right)=\left|B_{1}\right|-\frac{p^{e(d-1)}-1}{p^{e}-1}=p^{e(d-1)}$. Hence, $\operatorname{val}(\Gamma) \geq \operatorname{val}\left(\left[B_{1}, B_{2}\right]\right) \geq$ $\min \left\{\frac{p^{e(d-1)}-1}{p^{e}-1}, p^{e(d-1)}\right\}$, the minimal one of $\frac{p^{e(d-1)}-1}{p^{e}-1}$ and $p^{e(d-1)}$. By assumption, we have

$$
\min \left\{\frac{p^{e(d-1)}-1}{p^{e}-1}, p^{e(d-1)}\right\} \leq \operatorname{val}(\Gamma)<p_{1} \leq|\mathrm{Out}(T)| \leq 2 e\left(d, p^{e}-1\right)
$$

which is a contradiction.

\section{Soluble case}

Let $\Gamma, R, G$ satisfy Hypothesis 3.1. In this section, we investigate the case where $G$ is soluble.

Lemma 4.1 Suppose $G$ is soluble. Then $\hat{R}$ is normal in $G$.

Proof Let $\mathrm{F}$ be the Fitting subgroup of $G$. If a prime $p|| \mathrm{F} \mid$, then $1 \neq \mathrm{F}_{p} \triangleleft G$, by Lemma 3.1(2), $\mathrm{F}_{p} \cap \hat{R} \neq 1$ and so $p$ divides $|\hat{R}|=n$. Then since $\mathrm{F}$ is nilpotent, we conclude

$$
\mathrm{F}=\mathrm{O}_{p_{1}}(G) \times \mathrm{O}_{p_{2}}(G) \times \cdots \times \mathrm{O}_{p_{s}}(G)
$$

where $\mathrm{O}_{p_{i}}(G)$ denotes the largest normal $p_{i}$-subgroup of $G$.

For each $i \in\{1,2, \ldots, s\}$, recall that $\left|G_{p_{i}}\right|=p_{i}$, we have $\mathrm{O}_{p_{i}}(G) \leq \mathbb{Z}_{p_{i}}$, so F is a cyclic group. Then by Lemma $2.1, C_{G}(\mathrm{~F})=\mathrm{F}$, and $G / \mathrm{F}=G / C_{G}(\mathrm{~F}) \leq \operatorname{Aut}(\mathrm{F})$ is abelian. Further, if some $\mathrm{O}_{p_{i}}(G) \neq 1$, by Lemma 3.1(2), $\mathrm{O}_{p_{i}}(G) \cap \hat{R} \neq 1$, so $\mathrm{O}_{p_{i}}(G) \subseteq \hat{R}$. It follows $\mathrm{F} \subseteq \hat{R}$. Now, as $G / \mathrm{F}$ is abelian and $\hat{R} / \mathrm{F} \leq G / \mathrm{F}$, we obtain $\hat{R} / \mathrm{F} \triangleleft G / \mathrm{F}$, and so $\hat{R} \triangleleft G$.

Let 1 denote the vertex of $\Gamma$ corresponding to the identity element of $R$. By Lemmas 4.1, $\hat{R} \triangleleft G$, then Lemma 2.4 implies $G_{1} \leq \operatorname{Aut}(R, S) \leq \operatorname{Aut}(R)$. We therefore need information of the automorphism group $\operatorname{Aut}(R)$. 
If $R \cong \mathbb{Z}_{n}$ is cyclic, then $\operatorname{Aut}(R) \cong \mathbb{Z}_{n}^{*}$ is abelian. Suppose $R$ is not cyclic. Since $R$ is of square-free order, we may write $R=\langle a\rangle \times(\langle b\rangle:\langle c\rangle)$ with $Z(\langle b\rangle:\langle c\rangle)=1$, where $Z(\langle b\rangle:\langle c\rangle)$ denotes the center of the group $\langle b\rangle:\langle c\rangle$.

Lemma 4.2 Let $R=\langle a\rangle \times(\langle b\rangle:\langle c\rangle) \cong \mathbb{Z}_{h} \times\left(\mathbb{Z}_{t}: \mathbb{Z}_{l}\right)$ with $Z(\langle b\rangle:\langle c\rangle)=1$. Then $\operatorname{Aut}(R) \cong \mathbb{Z}_{h}^{*} \times\left(\mathbb{Z}_{t}: \mathbb{Z}_{t}^{*}\right)$, and further, each automorphism $\sigma \in \operatorname{Aut}(R)$ has the form:

$$
\sigma: a \rightarrow a^{x}, b \rightarrow b^{y}, c \rightarrow b^{z} c, \text { where }(x, h)=(y, t)=1,0 \leq z \leq t-1 .
$$

In particular, an element of $R$ with order $l$ is not conjugate to its inverse under Aut $(R)$, and if $b^{i} c^{j}$ with $i, j$ integers is of order $l$, then $b^{i} c^{j}$ is conjugate to $c^{j}$ under $\operatorname{Aut}(R)$.

Proof Since $R$ is of square-free order, the subgroups $\langle a\rangle,\langle b\rangle$ and $\langle b, c\rangle$ are characteristic subgroups of $R$, so we may suppose $\sigma: a \rightarrow a^{x}, b \rightarrow b^{y}, c \rightarrow b^{z} c^{m}$, where $(x, h)=(y, t)=1$. Since $c$ normalizes $\langle b\rangle, b^{c}=b^{r}$, with $r^{l} \equiv 1(\bmod t)$. Then $\left(b^{y}\right)^{c^{m}}=b^{y r^{m}}$, and $\left(b^{y}\right)^{c^{m}}=\sigma(b)^{\sigma(c)}=\sigma\left(b^{c}\right)=\sigma\left(b^{r}\right)=b^{y r}$, so $y r^{m} \equiv$ $y r(\bmod t)$, and consequently $r^{m-1} \equiv 1(\bmod t)$. It follows $r^{(l, m-1)} \equiv 1(\bmod t)$. If $(l, m-1)$ is a proper divisor of $l$, then $c^{(l, m-1)} \neq 1$ is in the center of the group $\langle b\rangle:\langle c\rangle$, a contradiction. Thus, $l \mid(m-1)$, and $\sigma(c)=b^{z} c$.

Now, let

$$
\begin{aligned}
& M=\left\langle\sigma: a \rightarrow a^{x}, b \rightarrow b, c \rightarrow c \mid(x, h)=1\right\rangle \\
& N=\left\langle\sigma: a \rightarrow a, b \rightarrow b, c \rightarrow b^{z} c \mid 0 \leq z \leq t-1\right\rangle, \\
& K=\left\langle\sigma: a \rightarrow a, b \rightarrow b^{y}, c \rightarrow c \mid(y, t)=1\right\rangle
\end{aligned}
$$

Then it is easy to show that $\operatorname{Aut}(R)=M \times(N: K) \cong \mathbb{Z}_{h}^{*} \times\left(\mathbb{Z}_{t}: \mathbb{Z}_{t}^{*}\right)$.

Suppose $x \in R$ is of order $l$. Then $x=b^{i} c^{j}$ for some integers $i$ and $j$. Since $l$ is odd and $\left(b^{i} c^{j}\right)^{-1}=b^{i^{\prime}} c^{l-j}$ for some integer $i^{\prime}$, the above discussion shows $x$ is not conjugate to $x^{-1}$ under Aut $(R)$. Since $\left\langle b^{i} c^{j}\right\rangle$ and $\langle c\rangle$ are Hall subgroups of order $l$ of $R$, they are conjugate in $R$, it follows that $b^{i} c^{j}$ is conjugate to $c^{j}$ under $\operatorname{Aut}(R)$.

With Lemmas 4.1 and 4.2, we may here determine the graphs $\Gamma$ for the case $G$ is soluble. We remark, the full automorphism group Aut $\Gamma$ in the case will be determined in Lemma 6.4 since more results of general cases will be needed.

Lemma 4.3 Assume $G$ is soluble. Then $\Gamma$ is a $G$-normal Cayley graph of $R$, and further, one of the following holds:

1. $R=\langle a\rangle$ is cyclic, $S=a^{H}$ and $\Gamma$ is $(\hat{R}: H)$-arc-regular, where $H=\left\langle G_{\mathbf{1}}, \tau\right\rangle \leq$ Aut $(R)$ with $\tau$ mapping a to $a^{-1}$.

2. $R=\langle a\rangle \times(\langle b\rangle:\langle c\rangle) \cong \mathbb{Z}_{h} \times\left(\mathbb{Z}_{t}: \mathbb{Z}_{l}\right)$ with $Z(\langle b\rangle:\langle c\rangle)=1, S=$ $\left\{a c^{z},\left(a c^{z}\right)^{-1}\right\}^{K}$ with $(z, l)=1$ and $K=G_{1} \leq \mathbb{Z}_{h}^{*} \times \mathbb{Z}_{t}^{*}$ a subgroup of $\operatorname{Aut}(R)$, $\operatorname{val}(\Gamma)=2\left|G_{\mathbf{1}}\right|$, and $\Gamma$ is $G$-half-transitive and $G$-edge-regular.

Proof By Lemma 4.1, $\Gamma$ is a $G$-normal edge-transitive Cayley graph of $R$, so $G_{\mathbf{1}} \leq$ Aut $(R, S)$ has at most two orbits on $\Gamma(\mathbf{1})=S$, hence $S=\left\{s, s^{-1}\right\}^{G_{1}}$ for some $s \in S$ (see Construction 2.5). In particular, elements in $S$ have the same order. As $\Gamma$ is connected, $\langle S\rangle=R, G_{1}$ acts faithfully on $S$ since $G_{1} \leq \operatorname{Aut}(R)$. 
1. Suppose $R=\langle a\rangle \cong \mathbb{Z}_{n}$ is cyclic. Then, $s$ is of order $n$ and conjugate to $a$ under $\operatorname{Aut}(R)$, as Cay $(R, S) \cong \operatorname{Cay}\left(R, S^{\phi}\right)$ for each $\phi \in \operatorname{Aut}(R)$, we may suppose, upon the isomorphism, $s=a$. Since $R$ is cyclic, $R$ has an automorphism $\tau$ mapping $a$ to $a^{-1}$, and since $S=S^{-1}, \tau \in \operatorname{Aut}(R, S) \leq$ Aut $\Gamma$, hence $S=a^{H}$, where $H=\left\langle G_{\mathbf{1}}, \tau\right\rangle$. Further, since $H \leq \operatorname{Aut}(R) \cong \mathbb{Z}_{n}^{*}$ is abelian and acts faithfully and transitively on $S, H$ is regular on $S$, so $\operatorname{val}(\Gamma)=|S|=|H|$ and $\Gamma$ is $(\hat{R}: H)$ arc-regular.

2 Suppose $R$ is not cyclic. Then, we may write $R=\langle a\rangle \times(\langle b\rangle:\langle c\rangle) \cong \mathbb{Z}_{h} \times$ $\left(\mathbb{Z}_{t}: \mathbb{Z}_{l}\right)$ with $Z(\langle b\rangle:\langle c\rangle)=1$. Write $s=a^{x} b^{y} c^{z}$. For each $\sigma \in \operatorname{Aut}(G)$, by Lemma $4.2, \sigma(s) \in\left\langle a^{x}, b, c^{z}\right\rangle$ and so $S \subseteq\left\langle a^{x}, b, c^{z}\right\rangle$, by the connectivity of $\Gamma, G=\left\langle a^{x}, b, c^{z}\right\rangle$. It follows $(x, h)=(z, l)=1$ and $b^{y} c^{z}$ is of order $l$. By Lemma 4.2, $a$ is conjugate to $a^{x}$ under $\operatorname{Aut}(R)$, and $b^{y} c^{z}$ is conjugate to $c^{z}$ under $\operatorname{Aut}(R)$, we have $a^{x} b^{y} c^{z}$ is conjugate to $a c^{z}$ under $\operatorname{Aut}(R)$. Hence, upon the isomorphism, we may assume $s=a c^{z}$, and so $S=\left\{a c^{z},\left(a c^{z}\right)^{-1}\right\}^{K}$, where $K=G_{\mathbf{1}} \leq \operatorname{Aut}(R)$. Again by Lemma 4.2, there is no $\sigma \in \operatorname{Aut}(G)$ which maps $s$ to $s^{-1}, \Gamma$ is $G$-half-transitive. Recall $K \leq \operatorname{Aut}(R) \cong \mathbb{Z}_{h}^{*} \times\left(\mathbb{Z}_{t}: \mathbb{Z}_{t}^{*}\right)$ and $(|K|, n)=1$, we have $K \leq \mathbb{Z}_{h}^{*} \times \mathbb{Z}_{t}^{*}$ is abelian. Now, since $\left\langle s^{K}\right\rangle=\left\langle\left(s^{-1}\right)^{K}\right\rangle=R$, $K$ acts faithfully on $s^{K}$ and $\left(s^{-1}\right)^{K}$, so is regular. It follows that $\operatorname{val}(\Gamma)=2|K|$, and $\Gamma$ is $G$-edge-regular.

\section{Almost simple case}

A transitive permutation group is called quasiprimitive if its minimal normal subgroups are transitive. Quasiprimitive permutation groups which are almost simple and contain a transitive metacyclic subgroup of odd order are known, see [16]. Then, we easily deduce the following result.

Lemma 5.1 Let $X$ be an almost simple quasiprimitive permutation group on a set $\Omega$ and contains a regular metacyclic subgroup $K$ of odd order. Then either $\left(X, X_{\alpha}\right)=$ $\left(\mathrm{A}_{m}, \mathrm{~A}_{m-1}\right)$ or $\left(\mathrm{S}_{m}, \mathrm{~S}_{m-1}\right)$ with $m$ odd, or the triple $\left(X, K, X_{\alpha}\right)$ lies in Table 3 , where $p$ is a prime, $G\left(q^{d}\right)$ is a regular subgroup of $\Gamma \mathrm{L}\left(1, q^{d}\right)=\mathbb{Z}_{q^{d}-1}: \mathbb{Z}_{d}$, and $\alpha \in \Omega$.

Suppose $\Gamma, R$ and $G$ satisfy Hypothesis 3.1. In this section, we will work out the graphs $\Gamma$ for the case where $G$ is almost simple. Recall the socle of a group $G$, denoted by $\operatorname{soc}(G)$, is the product of all minimal normal subgroups of $G$.

First, the following lemma determines the candidates of the group $G$.

Lemma 5.2 Suppose $G$ is almost simple. Then, $G$ is quasiprimitive on $V \Gamma$, and the triple $\left(G, \hat{R}, G_{\alpha}\right)\left(\right.$ as $\left(X, K, X_{\alpha}\right)$ there) satisfies Lemma 5.1, where $\alpha \in V \Gamma$.

Proof Let $T=\operatorname{soc}(G)$. Then, $G=T . o$ with $o \leq \operatorname{Out}(T)$.

Assume, on the contrary, $T$ is intransitive on $V \Gamma$. Let $B=\alpha^{T}$ be an orbit of $T$ on $V \Gamma$. Then, $G_{B}^{B}$ is almost simple with socle $T^{B} \cong T$, and by Lemma 3.1(2), $\hat{R} \cap T$ is regular on $B$. That is, $G_{B}^{B}$ is an almost simple quasiprimitive permutation group on $B$ containing a regular metacyclic subgroup $\hat{R} \cap T$. By Lemma 5.1, either

(i) $\left(G_{B}^{B},\left(G_{B}^{B}\right)_{\alpha}\right)=\left(\mathrm{A}_{m}, \mathrm{~A}_{m-1}\right)$ or $\left(\mathrm{S}_{m}, \mathrm{~S}_{m-1}\right)$ with $m$ odd; or 
Table 3 All most simple quasiprimitive permutation groups containing a regular metacyclic subgroup of odd order

\begin{tabular}{lllll}
\hline Row & $X$ & $K$ & $X_{\alpha}$ & Conditions \\
\hline 1 & $\mathrm{~A}_{p} . o$ & $p: \frac{p-1}{2}$ & $\mathrm{~S}_{p-2} \times o$ & $o \leq 2, p \equiv 3(\bmod 4)$ \\
2 & $\mathrm{PSL}(d, q) . o$ & $G\left(q^{d}\right)$ & $P_{1} . o$ & $q=p^{e}, o \leq(d, q-1) \cdot e$ \\
3 & $\mathrm{PSL}(2, p) . o$ & $p: \frac{p-1}{2}$ & $\mathrm{D}_{(p+1) o}$ & $o \leq 2, p \equiv 3(\bmod 4)$ \\
4 & $\mathrm{PSL}(2,11) . o$ & $11: 5$ & $\mathrm{~A}_{4} . o$ & $o \leq 2$ \\
5 & $\mathrm{PSL}(2,11)$ & 11 & $\mathrm{~A}_{5}$ & \\
6 & $\mathrm{PSL}(2,23)$ & $23: 11$ & $\mathrm{~S}_{4}$ & \\
7 & $\mathrm{PSL}(2,29)$ & $29: 7$ & $\mathrm{~A}_{5}$ & \\
8 & $\mathrm{PSL}(2,59)$ & $59: 29$ & $\mathrm{~A}_{5}$ & \\
9 & $\mathrm{PSL}(5,2)$ & $31: 5$ & $2^{6}:\left(\mathrm{S}_{3} \times \mathrm{PSL}(3,2)\right)$ & \\
10 & $\mathrm{M}_{11}$ & 11 & $\mathrm{M}_{10}$ & \\
11 & $\mathrm{M}_{11}$ & $11: 5$ & $\mathrm{M}_{9} \cdot 2$ & \\
12 & $\mathrm{M}_{23}$ & 23 & $\mathrm{M}_{22}$ & \\
13 & $\mathrm{M}_{23}$ & $23: 11$ & $\mathrm{M}_{21} .2,2^{4} . \mathrm{A}_{7}$ & \\
\hline
\end{tabular}

(ii) $\left(G_{B}^{B}, R \cap T,\left(G_{B}^{B}\right)_{\alpha}\right)$ lies in Table 3 .

Since $T$ is intransitive on $V \Gamma$, there is a prime $p_{i} \in \pi$ such that $p_{i} \chi\left|T: T_{\alpha}\right|$. Then, since $T_{\alpha} \leq G_{\alpha}$ is a $\pi^{\prime}$-subgroup, $p_{i} \backslash|T|$. It follows $p_{i}||$ Out $(T) \mid$ since $G \leq T$. Out $(T)$. Clearly, $T \neq \mathrm{A}_{m}$ with $m$ odd. Further, checking the candidates in Table 3 , the only possibility is $\left(T, T_{\alpha}\right)=\left(\operatorname{PSL}(d, q), P_{1}\right)$. By Lemma 3.2 , it is a contradiction.

Thus, $T$ is transitive on $V \Gamma$ and $G$ is quasiprimitive on $V \Gamma$, hence the triple $\left(G, \hat{R}, G_{\alpha}\right)$ satisfies Lemma 5.1.

The next lemma further determines the graphs $\Gamma$ in the almost simple case.

Lemma 5.3 Assume $G$ is almost simple with socle $T$. Then $\Gamma$ is $T$-edge-transitive and lies in Table 1.

Proof By Lemma 5.2, $\left(G, R, G_{\alpha}\right)$ satisfies Lemma 5.1. We analyze all the candidates there.

Suppose first $G$ is 2-transitive on $V \Gamma$. Then $\Gamma \cong \mathrm{K}_{n}$ is a complete graph, and by assumption, $n-1=\operatorname{val}(\Gamma)$ is less than the smallest prime divisor of $n, n$ is a prime, which give rise to examples as in Rows 1-6 of Table 1.

Suppose now $\left(T, T_{\alpha}\right)=\left(\mathrm{A}_{p}, \mathrm{~S}_{p-2}\right)$, as in Row 1 of Table 3. Then $|V \Gamma|=\mid T$ : $T_{\alpha} \mid=\frac{p(p-1)}{2}$, and the action of $G$ on $V \Gamma$ is permutation equivalent to the action of $G$ acting on $\Omega^{\{2\}}$ (the set of 2-subsets of $\Omega$ ), induced by the natural action of $G$ on $\Omega:=\{1,2, \ldots, p\}$ of degree $p$. Identifying $V \Gamma$ with $\Omega^{\{2\}}$, and let $\alpha=\{1,2\}$. Then, for $\beta=\{i, j\} \in \Omega^{\{2\}}$, if both $i$ and $j$ are not equal to 1 or 2 , then $T_{\alpha \beta} \cong \mathrm{S}_{p-4} \cdot \mathbb{Z}_{2}$ and $\left|T_{\alpha}: T_{\alpha \beta}\right|=\frac{(p-2)(p-3)}{2}$. On the other hand, if one of $i$ and $j$ equals 1 or 2 , then 
$T_{\alpha \beta} \cong \mathrm{A}_{p-3}$ and $\left|T_{\alpha}: T_{\alpha \beta}\right|=2(p-2)$. Hence, $\operatorname{val}(\Gamma) \geq \min \left\{2(p-2), \frac{(p-2)(p-3)}{2}\right\}$ is larger than the smallest prime divisor of $|V \Gamma|=\frac{p(p-1)}{2}$. This is a contradiction.

Suppose $\left(G, G_{\alpha}\right)=\left(\operatorname{PSL}(2, p) . o, \mathrm{D}_{(p+1) o}\right)$ with $o \leq \mathbb{Z}_{2}$, as in Row 3 of Table 3 . Write $G_{\alpha}=\langle a\rangle:\langle b\rangle$ and let $\beta \in \Gamma(\alpha)$. If $a^{i}$ with order $o\left(a^{i}\right) \geq 3$ lies in $G_{\alpha \beta}$, then $\left\langle a^{i}\right\rangle$ is normal in both $G_{\alpha}$ and $G_{\beta}$, note that $G_{\alpha} \cong \mathrm{G}_{\beta}$ are maximal subgroup of $G$, it follows $G_{\beta}=N_{G}\left(\left\langle a^{i}\right\rangle\right)=G_{\alpha}$. In particular, $G_{\alpha}$ fix $\beta$. Since $G$ acts edge-transitively on $\Gamma$, we obtain $\operatorname{val}(\Gamma)=2$, so $\Gamma$ is a cycle and Aut $\Gamma$ is a dihedral group, a contradiction. Thus, $G_{\alpha \beta} \leq \mathrm{D}_{4}$. If $G_{\alpha \beta}=1$, then $\operatorname{val}(\Gamma) \geq\left|G_{\alpha}: G_{\alpha \beta}\right| \geq p+1$ is bigger than the smallest prime divisor of $|V \Gamma|=\frac{p(p-1)}{2}$, which is not the case. Hence, $G_{\alpha \beta} \cong \mathbb{Z}_{2}$ or $\mathrm{D}_{4}$. Assume $G=\operatorname{PSL}(2, p)$. Then, $G_{\alpha} \cong \mathrm{D}_{p+1}$, and $\operatorname{val}(\Gamma) \in\left\{\frac{p+1}{4}, \frac{p+1}{2}, p+1\right\}$. By the restriction of $\operatorname{val}(\Gamma)$, the only possibility is $\operatorname{val}(\Gamma)=\frac{p+1}{4}$ with $\frac{p-1}{2}$ a prime. If $G=\operatorname{PGL}(2, p)$, then $G_{\alpha} \cong \mathrm{D}_{2(p+1)}$, and $\operatorname{val}(\Gamma) \geq \frac{p+1}{2}$ is bigger than the smallest prime divisor of $\frac{p(p-1)}{2}$, no graph $\Gamma$ exists in the case.

For the remaining candidates, $\Gamma$ is always of order a product of two distinct primes (note that for the case $n$ is a prime, $G$ is always 2-transitive which has been discussed above). Edge-transitive graphs of order a product of two distinct primes were classified in $[26,27]$. Checking the list there, $\Gamma$ lies in Rows 7-10 of Table 1.

Finally, for Rows 1-6 of Table $1, \Gamma$ is always a complete graph and $T$ is 2 -transitive on $V \Gamma$, so $\Gamma$ is $T$-arc-transitive. For the other candidates in Table 1, one easily see that $T_{\alpha}$ has at most two orbits on $\Gamma(\alpha), \Gamma$ is $T$-edge-transitive.

\section{Proofs of Theorem 1.1 and Corollary 1.2}

With outcomes in Sects. 3, 4 and 5, we will complete the proofs of Theorem 1.1 and Corollary 1.2 in this section.

Assume $\Gamma, R, G$ satisfy Hypothesis 3.1. Let $M$ be the soluble radical of $G$, namely, the largest soluble normal subgroup of $G$. Then, $M$ is a characteristic subgroup of $G$. Recall that $|R|=n=p_{1} p_{2} \cdots p_{s}$ with $p_{1}<p_{2}<\cdots<p_{s}$ primes and $\pi=\left\{p_{1}, p_{2}, \ldots, p_{s}\right\}$.

We first consider the trivial soluble radical case.

Lemma 6.1 Assume $M=1$ and $G$ is not almost simple. Then no graph $\Gamma$ exists.

Proof Let $N=\operatorname{soc}(G)$. Since $M=1$, by Lemma 3.1(1), each minimal normal subgroup of $G$ is nonabelian simple, then Lemma 3.1(4) implies

$$
N:=T_{1} \times T_{2} \times \cdots \times T_{l}, \quad N_{\alpha}=\left(T_{1}\right)_{\alpha} \times\left(T_{2}\right)_{\alpha} \times \cdots \times\left(T_{l}\right)_{\alpha},
$$

where $l \geq 2$ and each $T_{i}$ is nonabelian simple and normal in $G$. It then follows from [10, Theorem 4.3A] that $T_{1}, T_{2}, \ldots, T_{l}$ are the only minimal normal subgroups of $G$. Further, since $T_{i}$ is not isomorphic to $T_{j}$ for $i \neq j$ by Lemma 3.1(3), we further conclude $T_{1}, T_{2}, \ldots, T_{l}$ are characteristic subgroups of $G$. Noting that the centralizer $C_{G}(N)=1$, we obtain $G \leq \operatorname{Aut}(N)=\left(T_{1}\right.$.Out $\left.\left(T_{1}\right)\right) \times \cdots \times\left(T_{l}\right.$.Out $\left.\left(T_{l}\right)\right)$.

Assume $N$ is intransitive on $V \Gamma$. Then $n \backslash|N|$, as $n|| G \mid$, there exists some $p_{i} \in \pi$ such that $p_{i}|| \operatorname{Out}\left(T_{i}\right) \mid$. Let $B=\alpha^{T_{i}}$, an orbit of $T_{i}$ on $V \Gamma$. By Lemma 3.1(2), $T_{i} \cap \hat{R}$ 
is regular on $B$, so $T_{i}^{B} \cong T_{i}$ is quasiprimitive containing a regular metacyclic subgroup $T_{i} \cap \hat{R}$, and hence satisfies Lemma 5.1. Then since $p_{i}||$ Out $\left(T_{i}\right) \mid$, the only possibility is $\left(T_{i},\left(T_{i}\right)_{\alpha}\right)=\left(\operatorname{PSL}(d, q), P_{1}\right)$. However, by Lemma 3.2, it is a contradiction.

Therefore, $N$ is transitive on $V \Gamma$, and $N \supseteq \hat{R}$ by Lemma 3.1(2). Now $\Sigma:=$ Cay $\left(R,\left\{s, s^{-1}\right\}^{N_{1}}\right)$ with $s \in S$ is a subgraph of $\Gamma$ and is $N$-edge-transitive. By Lemma $2.8, \Sigma \cong \Sigma_{1} \times \Sigma_{2} \times \cdots \times \Sigma_{l}$, where $\Sigma_{i}$ is a fused-orbital of $T_{i}$ on $\Omega_{i}:=\left[T_{i}\right.$ : $\left(T_{i}\right)_{\alpha}$ ] by coset action and is $T_{i}$-edge-transitive for $i=1,2, \ldots, l$. Recall $\left(T_{i},\left(T_{i}\right)_{\alpha}\right)$ satisfies Lemma 5.1. If $\left(T_{i},\left(T_{i}\right)_{\alpha}\right)=\left(\operatorname{PSL}(2,11), \mathrm{A}_{4}\right)$ for some $i$, then $\left|\alpha^{T_{i}}\right|=\mid T_{i}$ : $\left(T_{i}\right)_{\alpha} \mid=55$ divides $n$, so $p_{1} \leq 5$ and $\operatorname{val}(\Gamma)=2$ or 4 . The former case is not possible as the full automorphism group of a cycle is soluble. If $\operatorname{val}(\Gamma)=4$, by the classification of tetravalent edge-transitive Cayley graphs of square-free order given in [17], each such graph does no admit an edge-transitive graph which has more than one nonabelian normal subgroups, a contradiction. Hence $\left(T_{i},\left(T_{i}\right)_{\alpha}\right) \neq\left(\operatorname{PSL}(2,11), \mathrm{A}_{4}\right)$ for each $i$. Now, checking the candidates in Lemma 5.1, $\left(T_{i}\right)_{\alpha}$ is always maximal in $T_{i}$, so $T_{i}$ is primitive on $\Omega_{i}$, and hence $\Sigma_{i}$ is connected by Lemma 2.7. Further, as $T_{i} \cap \hat{R}$ is regular on $\Omega_{i}$ by Lemma 3.1(2), one easily see that $\Sigma_{i}$ satisfies Lemma 5.3, and consequently, lies in Table 1 . Since $\operatorname{val}(\Sigma)=\operatorname{val}\left(\Sigma_{1}\right) \cdot \operatorname{val}\left(\Sigma_{2}\right) \cdots \operatorname{val}\left(\Sigma_{l}\right)$ and $l \geq 2$, by checking the valency, we always have $\operatorname{val}(\Gamma) \geq \operatorname{val}(\Sigma)>p_{1}$, which is a contradiction.

To treat the nontrivial soluble radical case, we need the following lemma.

Lemma 6.2 Assume $G$ is insoluble. Then $G=(M \times T)$.o, where $T$ is a nonabelian simple group and $o \leq \operatorname{Out}(T)$.

Proof We prove Lemma 6.2 by using induction on the order of $\Gamma$. If $M=1$, Lemma 6.2 is true by Lemma 6.1.

Assume $M \neq 1$. Let $\mathrm{F}$ be the Fitting subgroup of $M$. Then $\mathrm{F}$ is clearly also the Fitting subgroup of $G$. Since $M \neq 1$, by Lemma $2.1, \mathrm{~F} \neq 1$ and $C_{M}(\mathrm{~F}) \leq \mathrm{F}$. As $\mathrm{F}$ is nilpotent, arguing similarly as in the proof of Lemma 4.1 , we obtain $\mathrm{F} \leq \mathbb{Z}_{n}$ is a subgroup of $R$, and so $C_{M}(\mathrm{~F})=\mathrm{F}$. If $\mathrm{F} \cong \mathbb{Z}_{n}$, then $\mathrm{F}$ is regular on $V \Gamma, \Gamma$ is a $G$-normal Cayley graph of F, by Lemma $2.4, G \leq N_{\text {Aut } \Gamma}(\hat{\mathrm{F}}) \leq \hat{\mathrm{F}}: \operatorname{Aut}(\mathrm{F}) \cong \mathbb{Z}_{n}: \mathbb{Z}_{n}^{*}$ is soluble, a contradiction. Thus $\mathrm{F}<\mathbb{Z}_{n}$ is intransitive on $V \Gamma$. Let $K$ be the kernel of $G$ acting on $V \Gamma_{\mathrm{F}}$, the set of F-orbits on $V \Gamma$. Then $\mathrm{F} \triangleleft K$, and for $B \in V \Gamma_{\mathrm{F}}, F$ is regular on $B$, by [10, Corollary $4.2 \mathrm{~B}], K^{B} \leq N_{\operatorname{Sym}(B)}(F)=F$ : Aut $(F)$ is soluble. It then follows from Lemma 2.3(2) that $K \leq \prod_{B \in V \Gamma_{F}} K^{B}$ is soluble. In particular, $K \leq M$.

Now $\Gamma_{\mathrm{F}}$ is a connected $G / K$-edge-transitive graph. Note that $G / K$ has a transitive subgroup $\hat{R} K / K$ on $V \Gamma_{\mathrm{F}}$. Since $|\hat{R} K / K|=|\hat{R} /(\hat{R} \cap K)|$ is a divisor of $n$ and $\operatorname{val}\left(\Gamma_{\mathrm{F}}\right) \leq \operatorname{val}(\Gamma)<p_{1}$, we have $\left(\left|(\hat{R} K / K)_{\delta}\right|, n\right)=1$ for $\delta \in V \Gamma_{\mathrm{F}}$, so $(\hat{R} K / K)_{\delta}=$ 1 and $\hat{R} K / K$ is regular on $V \Gamma_{\mathrm{F}}$, that is, $\Gamma_{\mathrm{F}}$ is a Cayley graph of $\hat{R} K / K$. Therefore, the triple $\left(\Gamma_{\mathrm{F}}, \hat{R} K / K, G / K\right)$ satisfies Hypothesis 3.1. Since the soluble radical of $G / K$ is $M / K$, by induction, we have $G / K=(M / K \times T)$.o, and in turn, $G \cong M . T . o$, where $T$ is nonabelian simple and $o \leq \operatorname{Out}(T)$. In particular, $T$ is the unique insoluble composition factor of $G$.

Let $C=C_{G}(\mathrm{~F})$. Noting that $\mathrm{F}$ a normal Hall subgroup of $G$ (and so of $C$ ), by SchurZassenhaus theorem, $\mathrm{F}$ has a complementary subgroup in $C$, say $H$, hence $C=\mathrm{F} \times H$. 
Since $G / C \leq \operatorname{Aut}(\mathrm{F})$ is abelian, $C$ is insoluble, so is $H$. Let $M_{1}$ be the soluble radical of $H$, and let $\mathrm{F}_{1}$ be the Fitting subgroup of $M_{1}$. Since $\mathrm{F}_{1}$ is characteristic in $M_{1}, M_{1}$ is characteristic in $H$ and $H$ is normal in $G$, we have $\mathrm{F}_{1}$ is normal in $G$. If $M_{1} \neq 1$, by Lemma $2.1, \mathrm{~F}_{1} \neq 1$. It follows $\left\langle\mathrm{F}, \mathrm{F}_{1}\right\rangle=\mathrm{F} \times \mathrm{F}_{1}>\mathrm{F}$ is a nilpotent normal subgroup of $G$, a contradiction. Hence $M_{1}=1$. Now, as $G$ has unique insoluble composition factor $T$, it easily follows $\operatorname{soc}(H)=T$. Thus, $\langle M, T\rangle=M \times T \triangleleft G$ and $G=(M \times T) . o$.

With Lemma 6.2, we here determine the corresponding graphs $\Gamma$ when $M \neq 1$.

Lemma 6.3 Assume $G$ is insoluble and $M \neq 1$. Then $\Gamma$ satisfies part (5) of Theorem 1.1.

Proof By Lemmas 6.2 and 3.1, $G=(M \times T) . o$ and $M$ is intransitive on $V \Gamma$. Let $K$ by the kernel of $G$ acting on $V \Gamma_{M}$. Then $M \triangleleft K \triangleleft G, \alpha^{M}=\alpha^{K}$ and $|M|_{\pi}=|K|_{\pi}$ by Lemma 3.1(2). If $M<K$, as $K / M \triangleleft G / M \cong T$.o, we have $|M|_{\pi}|T|_{\pi}$ divides $|K|_{\pi}$, implying $|T|_{\pi}=1$, which is a contradiction again by Lemma 3.1(2). Hence $K=M$. Moreover, arguing similarly as in the proof of Lemma 6.2, we have $\hat{R} M / M$ is regular on $V \Gamma_{M}$, it follows that $\Gamma_{M}$ is a connected $G / M$-edge-transitive Cayley graph of $\hat{R} M / M$, and satisfies Hypothesis 3.1.

Since $G / M$ is almost simple with socle isomorphic to $T$ (for convenience, we also use $T$ to denote the socle of $G / M$ ), by Lemma 5.3, $\Gamma_{M}$ is $T$-edge-transitive, and in turn, $\Gamma$ is $X$-edge-transitive with $X=M \times T$. By Lemma 3.1(4), $X_{\alpha}=M_{\alpha} \times T_{\alpha}$, and then by Lemma 2.8, $\Gamma \cong \Delta \times \Sigma$, where $\Delta$ is a connected $M$-edge-transitive graph and $\Sigma$ is a connected $T$-edge-transitive graph. Further, by Lemma 3.1(3), $\hat{R} \cap M$ is regular on $V \Delta$ and $\hat{R} \cap T$ is regular on $V \Sigma$, hence $\Delta$ satisfies Lemma 4.3, and $\Sigma$ lies in Table 1 by Lemma 5.3. Now, since $\operatorname{val}(\Gamma)=\operatorname{val}(\Delta) \cdot \operatorname{val}(\Sigma)<p_{1}$, checking the valency, there are only two possibilities:

(a) $\operatorname{val}(\Delta)=2, T=\operatorname{PSL}(2,23)$ and $\operatorname{val}(\Sigma)=4$.

(b) $\operatorname{val}(\Delta) \leq 4, T=\operatorname{PSL}(2,59)$ and $\operatorname{val}(\Sigma) \leq 12$.

For the former case, $\Delta$ is a cycle of length $m$ say and $M \cong \mathrm{D}_{2 m}, \Gamma$ satisfies part (5)(i) of Theorem 1.1. For case (b), $\operatorname{val}(\Gamma)=\operatorname{val}(\Delta) \cdot \operatorname{val}(\Sigma)$ is less than the smallest prime divisor of $|V \Delta|$ and 29, $\Gamma$ satisfies part (5)(ii) of Theorem 1.1.

We may now determine the full automorphism group Aut $\Gamma$ while $G$ is soluble.

Lemma 6.4 Let $\mathrm{A}=$ Aut $\Gamma$ and suppose $G$ is soluble. Then one of the following statements holds.

1. $\mathrm{A}=G$;

2. $\Gamma \cong \mathrm{K}_{n}$ with $n \geq 5$ a prime, $G \cong \mathbb{Z}_{n}: \mathbb{Z}_{\frac{n-1}{2}}$ or $\mathbb{Z}_{n}: \mathbb{Z}_{n-1}$ and $\mathrm{A}=\mathrm{S}_{n}$;

3. $R=\langle a\rangle \cong \mathbb{Z}_{n}$ with $n$ not a prime, $\tau \notin G_{1}$ and $\mathrm{A}=G:\langle\tau\rangle \cong G: \mathbb{Z}_{2}$, where $\tau \in \operatorname{Aut}(R)$ maps a to $a^{-1}$;

4. $R \cong \mathbb{Z}_{11}: \mathbb{Z}_{5}, G \cong \mathbb{Z}_{11}: \mathbb{Z}_{10}, \mathrm{~A} \cong \operatorname{PGL}(2,11)$ and $\Gamma$ is of valency 4 .

Proof Assume $\mathrm{A} \neq G$. Then $R<G<\mathrm{A}$, and $\Gamma$ is A-edge-transitive.

Suppose first A is soluble. By Lemma $4.3, \Gamma$ is a normal Cayley graph, and satisfies part (1) or (2) of Lemma 4.3. For part (1), $R$ is cyclic, and as $\tau \in \mathrm{A}_{\mathbf{1}}$, both $\hat{R}:\left\langle G_{\mathbf{1}}, \tau\right\rangle$ 
and $\mathrm{A}=\hat{R}: \mathrm{A}_{\mathbf{1}}$ are regular on the arc set of $\Gamma, \hat{R}:\left\langle G_{\mathbf{1}}, \tau\right\rangle=\hat{R}: \mathrm{A}_{\mathbf{1}}$. Since $\mathrm{A}_{1} \leq \operatorname{Aut}(R)$ is abelian, it follows $\tau \notin G_{1}$ and $\mathrm{A}=G:\langle\tau\rangle \cong G: \mathbb{Z}_{2}$ since $\mathrm{A} \neq G$. For part (2), both $G$ and A are regular on the edge set of $\Gamma$, so $\mathrm{A}=G$, which is a contradiction.

Suppose now A is insoluble. By Lemmas 5.3, 6.1 and 6.3, either $\Gamma$ lies in Table 1 or satisfies parts (5) of Theorem 1.1. Then since A has a soluble edge-transitive subgroup $G$ of $\Gamma$, either $\Gamma$ is a complete graph $\mathrm{K}_{n}$ with $n$ a prime, or $\mathrm{A}=\operatorname{PGL}(2,11)$, $G \cong \mathbb{Z}_{11}: \mathbb{Z}_{10}, R \cong \mathbb{Z}_{11}: \mathbb{Z}_{5}$ and $\Gamma$ is of valency 4 . For the former case, as $G$ is edge-transitive on $\Gamma, G$ is 2 -homogeneous of prime degree $n$, hence $G \cong \mathbb{Z}_{n}: \mathbb{Z}_{\frac{n}{2}}$ or $\mathbb{Z}_{n}: \mathbb{Z}_{n-1}$ since $G$ is soluble.

Now, we are ready to prove Theorem 1.1 and Corollary 1.2.

Proof of Theorem 1.1 Let $\mathrm{A}=\mathrm{Aut} \Gamma$. Then $R<G \leq \mathrm{A}$ and $\Gamma$ is A-edge-transitive.

Assume first $G$ is soluble. If A is insoluble, then $G<\mathrm{A}$, by Lemma 6.4, part (1)(i) or part (3) of Theorem 1.1 holds. If $A$ is soluble, by Lemmas 4.3 and 6.4, $\Gamma$ is a normal Cayley graph of $R$, and either $\mathrm{A}=G: \mathbb{Z}_{2}$ and $R$ is cyclic or $\mathrm{A}=G$. It follows that $\Gamma$ satisfies part (1)(ii) or part (2) of Theorem 1.1.

Assume now $G$ is insoluble. Then A is insoluble. Let $M$ denote the soluble radical of A. If $M=1$, by Lemma 6.1, A is almost simple, then by Lemma 5.3, $\Gamma$ lies in Table 1, part (4) of Theorem 1.1 holds. If $M \neq 1$, by Lemma $6.3, \Gamma \cong \Delta \times \Sigma$ satisfies part (5) of Theorem 1.1. This completes the proof of Theorem 1.1.

Proof of Corollary 1.2 Since $\Gamma$ is a locally primitive Cayley graph, $\Gamma$ is arc-transitive, so $\Gamma$ satisfies parts (1) or (3)-(5) of Theorem 1.1. Let $\mathrm{A}=\operatorname{Aut} \Gamma$. Then $\mathrm{A}_{\alpha}$ with $\alpha \in V \Gamma$ is primitive on $\Gamma(\alpha)$.

If $\Gamma$ is arc-regular, as in part (1)(ii) of Theorem 1.1, then since val $(\Gamma)$ is even and $\mathrm{A}_{\alpha}$ is abelian, $\mathrm{A}_{\alpha}$ is not primitive on $\Gamma(\alpha)$, which is not the case. If $\Gamma$ lies in part (1)(i) or part (3) and (4), by the primitivity of $\mathrm{A}_{\alpha}$ acting on $\Gamma(\alpha), \Gamma$ is listed in Table 2. Finally, one easily shows the graphs in part (5) of Theorem 1.1 are not locally primitive.

\section{Proofs of Theorems 1.3 and 1.4}

The following number-theoretic result is known and easy to prove.

Lemma 7.1 For each positive integer $k \geq 2$, there are infinitely many primes $p$ such that $k \mid(p-1)$.

We finally prove Theorems 1.3 and 1.4.

Proof of Theorem 1.3 We first give a construction.

Construction 7.2 Choose distinct primes $p_{1}<p_{2}<\cdots<p_{s}$ such that $p_{1}>2 k+1$ and $2 k \mid\left(p_{i}-1\right)$ for $i=1,2, \ldots$, s. Set $n=p_{1} p_{2} \cdots p_{s}$ and let $R=\langle a\rangle$ be a cyclic group of order $n$. Then $\operatorname{Aut}(R) \cong \mathbb{Z}_{p_{1}-1} \times \mathbb{Z}_{p_{2}-1} \times \cdots \times \mathbb{Z}_{p_{s}-1}$ has a subgroup $H$ of order $2 k$ which contains the automorphism $\tau$ of $R$ mapping a to $a^{-1}$.

Let $S=a^{H}$ and set $\Gamma=\operatorname{Cay}(R, S)$. 
Since $\langle S\rangle=R, \Gamma$ is connected. Also, $\Gamma$ has a soluble arc-regular automorphism group $\hat{R}: H$, with valency $\operatorname{val}(\Gamma) \leq|H|=2 k \leq p_{1}-2 \leq n-2$, hence $\Gamma$ is not a complete graph and satisfies Theorem 1.1. By checking the list there, $\Gamma$ is an arc-regular normal Cayley graph of valency $2 k$. Further, by Lemma 7.1, the primes $p_{1}, p_{2}, \ldots, p_{s}$ above have infinitely many choices, Theorem 1.3 follows.

Proof of Theorem 1.4 We give the following construction.

Construction 7.3 Let $p_{1}<p_{2}<\cdots<p_{t}$ be primes such that $5 \neq p_{1}>2 k$ and $p_{1} k \mid\left(p_{i}-1\right)$ for $i=2, \ldots, t$. Write $m=p_{2} p_{3} \cdots p_{s}$. Then there is a center free metacyclic group $R:=\langle a\rangle:\langle b\rangle \cong \mathbb{Z}_{m}: \mathbb{Z}_{p_{1}}$, such that $\operatorname{Aut}(R) \cong \mathbb{Z}_{m}: \mathbb{Z}_{m}^{*}$ (see Lemma 4.2) contains a subgroup $K$ of order $k$ such that $\left\langle b^{K}\right\rangle=R$.

Let $S=\left\{b, b^{-1}\right\}^{K}$ and $\Gamma=\operatorname{Cay}(R, S)$.

Now, $\Gamma$ is connected since $\left\langle b^{K}\right\rangle=R$. Since $\operatorname{val}(\Gamma)=|S| \leq 2|K|<p_{1}$ and $\Gamma$ has an edge-transitive automorphism group $\hat{R}: K, \Gamma$ satisfies Theorem 1.1. Since $\hat{R}: H$ is soluble and $p_{1} \neq 5$, by Theorem 1.1, $\Gamma$ is an edge-regular normal metacirculant with valency $2 k$. Because the primes $p_{1}, p_{2}, \ldots, p_{t}$ above have infinitely many choices by Lemma 7.1, Theorem 1.4 follows.

Acknowledgements The authors are very grateful to the referee for the helpful comments.

\section{References}

1. Alspach, B.: Point-symmetric graphs and digraphs of prime order and transitive permutation groups of prime degree. J. Comb. Theory Ser. B 15, 12-17 (1973)

2. Alspach, B., Marušič, D., Nowitz, L.: Constructing graphs which are 1/2-transitive. J. Austral. Math. Soc. Ser. A 56(3), 391-402 (1994)

3. Biggs, N.: Algebraic Graph Theory, 2nd edn. Cambridge University Press, New York (1992)

4. Bouwer, A.E.: Vertex and edge-transitive but not 1-transitive graphs. Can. Math. Bull. 13, 231-237 (1970)

5. Cameron, P.J.: Finite permutation groups and finite simple groups. Bull. Lond. Math. Soc. 13, 1-22 (1981)

6. Chao, C.Y.: On the classification of symmetric graphs with a prime number of vertices. Trans. Am. Math. Soc. 158, 247-256 (1971)

7. Cheng, Y., Oxley, J.: On weakly symmetric graphs of order twice a prime. J. Comb. Theory Ser. B 42 , 196-211 (1987)

8. Conder, M.D.E., Marušič, D.: A tetravalent half-arc-transitive graph with non-abelian vertex stabilizer. J. Comb. Theory Ser. B 88, 67-76 (2003)

9. Ding, S.Y., Ling, B., Lou, B.G., Pan, J.M.: Pentavalent symmetric graphs of square-free order. Graphs Comb. 32, 2355-2366 (2016)

10. Dixon, J., Mortimer, B.: Permutation Groups. Springer, New York (1996)

11. Feng, Y.-Q., Kwak, J.H.: Constructing an infinite family of cubic 1-regular graphs. European J. Combin. 23(5), 559-565 (2002)

12. Frucht, R.: A one-regular graph of degree three. Can. J. Math. 4, 240-247 (1952)

13. Godsil, C.D.: On the full automorphism group of a graph. Combinatorica 1, 243-256 (1981)

14. Hammack, R., Imrich, W., Klavžar, S.: Edge-transitive products. J. Algebraic Combin. 43(4), 837-850 (2016)

15. Li, C.H.: On isomorphisms of connected Cayley graphs. Discrete Math. 178, 109-122 (1998)

16. Li, C.H., Pan, J.M., Song, S.J., Wang, D.J.: A characterization of a family of edge-transitive metacirculant graphs. J. Comb. Theory Ser. B 107, 12-25 (2014)

17. Li, C.H., Lu, Z.P., Wang, G.X.: The vertex-transitive and edge-transitive tetravalent graphs of squarefree order. J. Algebraic Combin. 42(1), 25-50 (2015) 
18. Li, C.H., Lu, Z.P., Wang, G.X.: On edge-transitive graphs of square-free order. Electr. J. Comb. 22(3), 3-25 (2015)

19. Li, C.H., Lu, Z.P., Wang, G.X.: Arc-transitive graphs of square-free order and small valency. Discrete Math. 339, 2907-2918 (2016)

20. Liu, G.X., Lu, Z.P.: On edge-transitive cubic graphs of square-free order. European J. Combin. 45, 41-46 (2015)

21. Marušič, D.: A family of one-regular graphs of valency 4. European J. Combin. 18(1), 59-64 (1997)

22. Marušič, D.: Recent developments in half-transitive graps. Discrete Math. 182, 219-231 (1998)

23. Marušič, D., Šparl, P.: One-regular graphs and half-transitive graphs of valency 4. European J. Combin. 19, 345-354 (1998)

24. Marušič, D.: On 2-arc-transitivity of Cayley graphs. J. Comb. Theory Ser. B 87, 162-196 (2003)

25. Pan, J.M.: Locally primitive Cayley graphs of dihedral groups. European J. Combin. 36, 39-52 (2014)

26. Praeger, C.E., Xu, M.Y.: Vertex-primitive graphs of order a product of two distinct primes. J. Comb. Theory Ser. B 59, 245-266 (1993)

27. Praeger, C.E., Wang, R.J., Xu, M.Y.: Symmetric graphs of order a product of two distinct primes. J. Comb. Theory Ser. B 58, 299-318 (1993)

28. Suzuki, M.: Group Theroy II. Springer, New York (1985)

29. Wang, R.J., Xu, M.Y.: A classification of symmetric graphs of order $3 p$. J. Comb. Theory Ser. B 58, 197-216 (1993)

30. Tutte, W.T.: Connectivity in Graphs. University of Toronto Press, Toronto (1966) 